\section{Neurotoxicity of $\beta$-amyloid}

SIR - The neuropathology of Alzheimer's disease is characterized, in part, by the deposition of abnormal protein aggregates called $\beta$-amyloid. The metabolism of the $\beta$-amyloid precursor protein has emerged as a central issue in pathogenesis because of the discovery of point mutations in its structural gene in some forms of early-onset familial Alzheimer's disease ${ }^{1}$.

Yoshikawa et al. ${ }^{2}$ extended our observations of $\beta$-amyloid precursor protein (APP)-mediated neurotoxicity, using the identical cell culture system

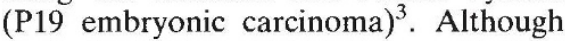
neurotoxicity of APP derivatives therefore seems to be well established, there are substantial differences between the results of the three laboratories that have reported on the overexpression of complementary DNA (cDNA) constructs for APP, or for parts of APP, in cultivated neuronal cells ${ }^{2-4}$. These various observations should also be put in the context of more recent results.

(1) Which products of APP metabolism are toxic to cultured neurons? Neurotoxicity of a carboxy-terminal fragment (spanning the $\beta$-amyloid peptide and cytoplasmic domains of APP) was originally reported by Yankner et al. ${ }^{4}$ by overexpression of its cDNA in differentiated neurons derived from a PC12 transformant. These authors did not observe neurotoxicity of protein products from a full-length cDNA construct for APP-695, however, in contrast to Yoshikawa et $a l .^{2}$. Using an antibody raised against the first 15 amino acids of the $\beta$-amyloid peptide, Yankner et al. observed highmolecular-mass $\quad(>110,000)$ protein aggregates on immunoblots from conditioned medium of a PC12 transformant bearing the DNA construct for the carboxy-terminal fragment. Fukuchi et $a .^{3}$ and Yoshikawa et al. ${ }^{2}$ did not observe such high-molecular-mass aggregates using antibodies against a cytoplasmic domain of APP in P19-derived neurons. Fukuchi et al. ${ }^{3}$ and Yoshikawa et al. $^{2}$ found multiple carboxy-terminal fragments, and Fukuchi et al. found that neuronal degeneration was associated with the generation of $M_{r} 16,000$ and 14,000 carboxy-terminal fragments.

(2) Is the neuronal specificity of the APP-derived cytotoxic moiety related to variations in processing or to variations in target cell specificity? Yoshikawa et al. did not find multiple carboxyterminal proteolytic fragments of APP in muscle cells derived from P19 transformants, suggesting that its proteolysis is different from that in neurons. We have also induced differentiation of our P19 transformants to muscle cells by treatment with dimethyl sulphoxide, and find that the proteolysis of APP in the muscle cells is similar to that in the neuronal cells, but that the 16,000 and 14,000 products did not affect the survival of muscle cells. This discrepancy may be due to differences in the choice of promoters with resultant differences in levels of transgene expression. Using several different cell lines 5 , promoter activities of a cytomegalovirus enhancer and chick $\beta$-actin promoter (which was used for the construction of our expression vector) are much higher than those of a SV40 promoter, which was used by Yoshikawa et al. Furthermore, when a DNA construct for the carboxy-terminal fragment was introduced into a morphologically heterogeneous neuroblastoma cell line (SK-N-SH) (consisting of neural and non-neural cell types), selective neurotoxicity of the fragment was observed ${ }^{6}$.

(3) Could neurotoxicity be mediated by a secreted $\beta$-amyloid peptide? Recent observations $^{7,8}$ have raised the possibility that neurotoxicity could be mediated by secreted, soluble $\beta$-amyloid peptide. To address this question, we have repeated the experiments of Fukuchi et al. $^{3,6}$, using conditioned media from stable transformants of P19 and SK-N-SH. Addition of conditioned media did not influence the neuronal differentiation of nontransfected P19 and SK-N-SH cells nor the survival of P19derived and SK-N-SH-derived neurons. We also did not observe secreted 16,000 and 14,000 fragments in conditioned media from the P19 transformants. We therefore propose that intracellular accumulations of the 16,000 and 14,000 fragments result in neural degeneration. Ken-ichiro Fukuchi

\section{Bryce Sopher}

George M. Martin

Department of Pathology,

University of Washington,

Seattle, Washington 98195, USA

YOSHIKAWA REPLIES - Yankner et al. ${ }^{4}$ and Fukuchi et al. ${ }^{3}$ found degenerative changes of neurons overexpressing a carboxy-terminal fragment of APP, which spans the $\beta / \mathrm{A} 4$ and cytoplasmic domains (the fragment lacks a signal sequence for secretion and membrane integration). Two years ago we demonstrated that a transient overexpression of the carboxy-terminal fragment, designated APP-C100, leads to a dense accumulation of amyloid-like fibrils near the nuclear membrane of COS cells ${ }^{9}$. Since then we have attempted to transfect APP-C100 cDNA into various cultured cell lines including PC12, P19, glioma and fibroblasts to establish stable transfectants, but we failed to obtain any clones expressing high levels of APPC100. Therefore, we assumed that an intracellular overproduction of APPC100 is extremely toxic to any type of cells (I suggest that the stable transfectants obtained in other laboratories ${ }^{3,4}$ express non-toxic levels of carboxyterminal fragments under undifferentiated conditions). We transfected human full-length APP cDNA into P19 embryonal carcinoma cells, which differentiate into post-mitotic neurons following retinoic acid treatment, to examine whether overexpressed APP undergoes aberrant processing to generate APPC100 or similar amyloidogenic fragments in differentiated neurons. We found severe degenerative changes of P19derived neurons which contained high amounts of potentially amyloidogenic carboxy-terminal fragments ${ }^{2}$.

More recently, we established human glioma cell lines (Bu-17) overexpressing full-length APP cDNA ${ }^{10}$. These transfectants accumulate carboxy-terminal fragments of APP and show degenerative changes when treated with chloroquine, a compound reducing lysosomal functions. Therefore, carboxy-terminal fragments of APP generated in the lysosomes may be toxic to non-neuronal cells as well as to neurons. We speculate that the susceptibility to overexpressed APP varies from cell to cell (even among neuronal subtypes) owing, at least in part, to the capability of metabolizing APP into non-toxic peptides. This may explain the absence of neurotoxicity of PC12 cells transfected with full-length APP cDNA ${ }^{4}$.

The carboxy-terminal fragments generated in the full-length APP cDNA transfectants are localized in the membrane fractions ${ }^{2}$. Neither conditioned media of these APP cDNA transfectants (P19, Bu-17) nor APP-C100 purified from cDNA-transfected cells showed neurotoxicity when added extracellularly (Y. Hayashi \& K. Y., unpublished observations). Therefore, these transfectants may be vulnerable to the intracellular amyloidogenic carboxy-terminal fragments.

The most likely idea is that extracellular amyloid deposits induce neuronal degeneration 'from outside'. Our cell models $2,9,10$ suggested a new concept, that intracellular amyloidogenic fragments kill neurons 'from inside'. In Alzheimer's disease and related disorders such as Down's syndrome, the

1. Goate, A. et al. Nature 349, 704-706 (1991)

2. Yoshikawa, K., Aizawa, T. \& Hayashi, Y. Nature 359. $64-67$ (1992).

3. Fukuchi, K. et al. Biochem biophys. Res. Commun 182, 165-173 (1992).

4. Yankner, B. A. et al. Science 245, 417--420 (1989)

5. Miyazaki, J. et al. Gene 79, 269-277 (1989).

6. Fukuchi, K. et al. Molec. Brain Res. 16, 37-46 (1992)

7. Seubert, P. et al. Nature 359, 325-327 (1992).

8. Haass, C. et al. Nature 359, 322-325 (1992).

9. Maruyama, K., Terakado, K., Usami, M. \& Yoshikawa K. Nature 347, 566-569 (1990).

10. Hayashi, Y., Kashiwagi, K. \& Yoshikawa, K. Biochem biophys. Res. Commun. 187, $1249-1255$ (1992). 